\title{
Unternehmerische Lösungsstrategien gegen Fachkräftemangel
}

Wegen der hohen Kosten der Herstellerschulungen erwerben die meisten Mitarbeiter die Kenntnisse in generativer Fertigung nach dem Prinzip „Learning by Doing“. Andere gern praktizierte Methoden, um zu qualifizierten Mitarbeitern zu gelangen, ist das Einstellen von externen Fachkräften aus dem Arbeitsmarkt oder „Guerilla-Recruiting“das Kapern von Mitarbeitern anderer Unternehmen wie z.B. der Anlagenhersteller. Unternehmen suchen vor allem Fachkräfte mit solider abgeschlossener handwerklicher und technischer Berufsausbildung. Beispiele solcher Berufe sind Werkzeugmacher oder Zahntechniker. Unternehmen, die parallel zu generativer Fertigung weiterhin eine traditionelle Fertigung betreiben, benötigen Mitarbeiter, welche auch die tradierten Prozesse verstehen und eine Brücke zwischen den ,alten“ Verfahren und AM schlagen können. Im Normalfall stellen die Unternehmen Personen mit handwerklicher oder technischer Ausbildung ein, um sie dann intern in AM zu schulen.

Unternehmen geben unterschiedliche Antworten auf die Frage, ob sie Schwierigkeiten haben, gute Mitarbeiterinnen und Mitarbeiter für die generative Fertigung zu finden und wie viel Zeit die Suche in Anspruch nimmt. Dabei stellen Region und Umfeld wichtige Faktoren dar, die darüber entscheiden, ob genügend gut ausgebildete Fachkräfte - zum Beispiel aus anderen Branchen - gewonnen werden können. Der Erfolg des Suchprozesses ist auch davon abhängig, ob das Fachkräftepotenzial in der Region genügend umfangreich ist oder ob zum Beispiel andere, große Unternehmen mit der Kapazität zu einer besseren Entlohnung der Beschäftigten genau diejenigen hoch qualifizierten Fachkräfte absorbieren, welche dann in der generativen Fertigung fehlen. 